\title{
Propuesta de atención para los servicios de psicoterapia en línea (telepsicoterapia) derivados del Covid-19 en México
}

\author{
Care proposal for online psychotherapy services \\ (telepsychotherapy) derived from the Covid-19 in Mexico
}

\author{
Raúl Gutiérrez Mercado ${ }^{l}$
}

\begin{abstract}
Citación: Gutiérrez M., R. (2020). Propuesta de atención para los servicios de psicoterapia en línea (telepsicoterapia) derivados del Covid-19 en México. Psicología y Salud, 30(1), 133-136.
\end{abstract}

\section{RESUMEN}

El presente documento pretende ser una propuesta de guía para aquellos profesionales de la salud mental que estén actualmente realizando terapia en línea, pero no cuentan con las directrices sobre cómo llevarla acabo de la manera más efectiva posible, acorde a algún documento científico que oriente, mas no necesariamente garantice, su efectividad, sobre todo en los momentos en que está vigente el periodo de cuarentena obligatoria en México debido al Covid-19.

Palabras clave: Covid-19; Telepsicoterapia; Fomento de la salud mental; Lineamientos y guía; Terapia en línea.

\begin{abstract}
This document is intended to be a proposal for to guide those mental health professionals who are currently practicing online therapy, but do not have the guidelienes or directions on how to carry them out in the most effective way posible, according to any scientific document that guides, but does not guarantee, the effectiveness of the sessions, specially in these moments where the mandatory quarantine period in Mexico, due to the Covid-19, is carried on.
\end{abstract}

Key words: Covid-19; Telepsychotherapy; Mental health support; Gudielines; Online therapy

$\int$

a emergencia sanitaria derivada del Covid-19, considerado desde el 30 de enero de 2020 como un problema de extrema preocupación internacional por la Organización Mundial de la Salud, y que desde el 11 de marzo fue calificada como una pandemia (Organización Panamericana de la Salud, 2020), ha llevado a muchos profesionales de la salud mental a practicar la terapia online. De manera paralela, y como es de esperarse, hay ya numerosos terapeutas que desean ayudar ofreciendo sus servicios por medio de la red o publicando recomendaciones sobre cómo practicar la psicoterapia en línea, también llamada telepsicoterapia, y también personas que incluso ofrecen capacitación al respecto (Reyes, 2020).

Ante una situación así, la tecnología resulta de considerable utilidad ya que permite atender al paciente a través de internet, aun en áreas geográficas muy lejanas, sin que la distancia sea una barrera para brindar la atención psicológica. El uso de estas tecnologías también hace posible la aproximación afectiva virtual con las personas cercanas, sean amigos, pareja o familiares. Este hecho ha provocado una nueva manera de relacionarnos con nosotros mismos y con la sociedad, instalándose desde hace algún tiempo en casi todas las áreas de la vida (Macías y Valero, 2018).

\footnotetext{
${ }^{1}$ Universidad Autónoma de Nayarit, Ciudad de la Cultura Amado Nervo, 63155 Tepic, Nay., México, tel. (311)211-88-01, correo electrónico: ragumer@msn.com. Artículo recibido el 22 de abril y aceptado el 26 de bril de 2020.
} 
La práctica de la telepsicología, definida como la provisión de servicios psicológicos utilizando tecnologías telecomunicacionales (American Psychological Association, 2013), ha venido ocurriendo de manera correspondiente a los avances tecnológicos y al surgimiento de nuevas técnicas de información y comunicación que facilitan el que dos o más personas interactúen por algún medio digital y electrónico. De igual forma, ha hecho posible la expansión de la psicología al consentir que todo aquel que cuenta con la accesibilidad necesaria atienda clases, cursos, conferencias, talleres, posgrados e incluso servicios de psicoterapia en línea, entre muchas otras actividades.

Entre los servicios de psicoterapia, la necesidad del terapeuta de flexibilizar sus servicios para poder asistir a sus consultantes (pacientes) -aunque no sea físicamente- se ha vuelto imperiosa (Gagliesi, 2020), más aún considerando el posible agravamiento de aquellos cuya sensibilidad emocional se ha agudizado por la crisis que atraviesa gran parte del mundo y, asimismo, por los efectos clínicos adversos que se experimentan por los periodos prolongados de aislamiento social.

Mediante una revisión sistemática, Macías y Valero (2018) detectaron que la terapia cognitivo-conductual es el modelo psicoterapéutico más socorrido, el tipo de tratamiento que más se aplica de manera online, y el que cuenta con más investigaciones disponibles en la literatura especializada internacional. Asimismo, detallan que los problemas clínicos que más se abordan son los trastornos traumáticos, los de la alimentación, del estado de ánimo, de ansiedad, de pánico, los obsesivo-compulsivos, las adicciones, el dolor crónico, los problemas familiares, el estrés en el cuidador y la depresión.

De primera importancia es también ofrecer y garantizar la accesibilidad a la telepsicoterapia del personal sanitario que se encuentra laborando en los hospitales, puesto que sus niveles de ansiedad, estrés, trauma, burnout, depresión y preocupación, entre muchas otras emociones o sensaciones adversas, pueden verse acentuados por la probable saturación del sistema médico-asistencial, tal como ha sucedido en países como China, Italia y España. Ante tales problemas, la salud mental del mencionado personal puede verse alterada aún más por la falta de insumos, instrumentos y materiales que pudiesen mitigar considerablemente la probabilidad de contagio, lo que merma sus funciones y reduce la calidad de los servicios de atención médica a las personas portadoras del virus.

Es por ello necesario implementar servicios efectivos de terapia en línea en este sector poblacional, y no únicamente de conferencias o reuniones virtuales psicoeducativas en grupo que numerosos profesionales llevan a cabo en estos momentos para coadyuvar al afrontamiento de la pandemia, actividades estas que, sin embargo, deben reconocerse como esenciales.

Bajo esta premisa, el uso de la telepsicoterapia haría posible ampliar desde su raíz la visión y el panorama de la problemática individual, contribuyendo así a evitar el deterioro de las estrategias psicológicas de afrontamiento que empleen los pacientes ante las crisis que puedan ocurrir, así como brindar al personal sanitario la contención emocional necesaria y el aumento del repertorio conductual adaptativo ante estas situaciones, lo que puede hacer, en consecuencia, que lleven a cabo su trabajo diario de una manera más adecuada y soporten mejor la pesada carga emocional o afectiva a la que están siendo sometidos.

A continuación, se sugieren ciertos lineamientos o requerimientos indispensables para aumentar dentro de lo posible la efectividad y calidad de las sesiones de terapia en línea, cuyos destinatarios son aquellos psicoterapeutas que disponen de las competencias para efectuarlas, y que actualmente se encuentren brindando servicios de telepsicoterapia.

Se aclara que en el presente documento se utilizan las palabras "consultante", "paciente" y "usuario que solicita la atención" como sinónimos para especificar a una misma persona, en este caso aquella que será atendida vía online por el psicoterapeuta.

\section{Principios éticos y legales}

Contrato terapéutico, consentimiento informado, privacidad y confidencialidad de la información.

- El psicoterapeuta cuenta con formación especializada de intervención en crisis, primeros auxilios psicológicos, abordaje de la 
ansiedad, estrés, depresión, burnout, trauma y afrontamiento de emociones adversas, tales como miedo, culpa, frustración y enojo, entre muchas otras que pudieran surgir. Dispone asimismo de las acreditaciones o documentos que prueban su calificación profesional y que le permiten el ejercicio de la terapia, preferentemente bajo el modelo terapéutico cognitivo-conductual. El paciente cuenta con el derecho de conocer dichas acreditaciones y documentos, mismas que avalan las competencias profesionales del psicoterapeuta del que está recibiendo sus servicios.

- Tanto el psicoterapeuta como el usuario que solicita la atención han llegado a un acuerdo mutuo para la intervención por medio de algún tipo de telecomunicación, estableciendo durante la misma el costo de las sesiones, su duración y frecuencia, la vía o medio de pago y la forma de llevar a cabo dicha intervención.

- Tanto el psicoterapeuta como el usuario que solicita la atención tienen la garantía y el derecho de que la sesión terapéutica no sea videograbada ni reproducida en forma alguna: audio, imagen, o ambas, a menos de que alguna de las dos partes lo solicite previamente, justificando sus motivos, y de que ambas lleguen al acuerdo y la autorización respectiva.

- Tanto el psicoterapeuta como el usuario que solicita la atención están conscientes de las ventajas y las desventajas de la intervención, así como de los posibles riesgos y beneficios de la misma. Si el consultante no los conoce, es deber y obligación del psicoterapeuta explicarlos de manera clara y concisa, hasta que sean correctamente comprendidos, asimilados y aprobados por aquel.

- Para el psicoterapeuta, el contenido abordado durante la sesión se mantiene bajo un estricto nivel de profesionalismo, de ética y de confidencialidad, acordes al Código ético del psicólogo de la Sociedad Mexicana de Psicología, violando únicamente la mencionada confidencialidad si se detecta que el consultante está en riesgo inminente de hacerse un daño hipotéticamente mortal a él mismo o a otros.

- En el caso de los consultantes menores de edad que soliciten la intervención, se deberá contar con la aprobación y el consentimiento de sus padres o tutores para llevar a cabo la telepsicoterapia, explicando tanto al consultante como a sus padres o tutores los puntos previamente mencionados, hasta llegar a los acuerdos pertinentes con respecto a la retribución económica y frecuencia de las sesiones, y siguiendo a la vez las mismas directrices estipuladas en el ya citado código ético del psicólogo.

\section{Aplicación y efectividad de la intervención}

Para aumentar el impacto, la efectividad del contenido de la sesión y la adquisición de información abordada de manera transaccional.

- Tanto el psicoterapeuta como el usuario que solicita la atención están ya familiarizados con el recurso o recursos tecnológicos de que se dispone como opciones para utilizarse.

- El psicoterapeuta y el usuario que solicita la atención seleccionan un lugar cómodo y privado dentro de su contexto (hogar, trabajo, oficina, etc.) en el que puedan permanecer durante el tiempo estipulado para la sesión, y sea así menos probable un fallo de la señal de internet o la interrupción de terceras personas.

- Tanto el psicoterapeuta como el usuario que solicita la atención están conscientes de los posibles distractores que pueden interferir durante la sesión (teléfonos celulares, televisión, ruidos ambientales, olores, otras personas, etc.) y hacen lo posible por evitarlos, maximizando así el efecto y resultados positivos del servicio.

- Al comienzo de la sesión vía online, tanto el psicoterapeuta como el paciente cierran en su dispositivo todas las ventanas de internet, pantallas o aplicaciones digitales que puedan ser un motivo de distracción. De igual manera, se cercioran de que puedan verse y escucharse adecuadamente antes de comenzar la sesión. 
- El psicoterapeuta deberá tener la pantalla visual del consultante maximizada en su computadora o dispositivo móvil, mientras que la suya estará minimizada; lo inverso será en la del consultante; es decir, su pantalla estará minimizada o hecha sustancialmente más pequeña, mientras que la del psicoterapeuta aparecerá maximizada, esto para evitar la distracción que pudiera generar el estarse viendo a sí mismos a través del medio de telecomunicación seleccionado.

- Al finalizar la sesión, el psicoterapeuta solicitará al paciente que valore su nivel de satisfacción con el servicio, medido por unidades subjetivas de bienestar de 0 a 10 o de 0 a 100, así como la justificación de dicha calificación, indagando también todos aquellos aspectos que se pueden mejorar para la siguiente sesión de telepsicoterapia acordada de mutuo acuerdo. El psicoterapeuta deberá prestar especial atención a estos puntos para ir mejorando la calidad de la intervención conforme transcurra el resto de las sesiones.

\section{CONCLUSIÓN}

El presente documento pretende ser una propuesta de guía para aquellos profesionales de la salud mental que estén actualmente realizando terapia en línea y no cuentan con las directrices o la dirección necesaria sobre cómo llevarlas a cabo del modo más efectivo posible y acorde a algún documento científico que oriente, aunque no garantice, la efectividad de la sesión, sobre todo en estos momentos de cuarentena obligatoria en México debida a la pandemia de Covid-19.

El presente autor no pretende de ninguna forma aseverar que los servicios de terapia en línea son o pudieran ser más efectivos que la modalidad presencial, dejando en claro únicamente que esta opción es una manera flexible de atender sectores poblacionales que tienen una mayor vulnerabilidad para experimentar intensos estados emocionales adversos.

Asimismo, como es de suponer, es consciente de que una gran cantidad de personas igualmente vulnerables no contarán con la accesibilidad necesaria para disponer de este servicio debido a las limitaciones socioeconómicas y geográficas que implica la interacción mediante una red de internet.

\section{REFERENCIAS}

American Psychological Association (2013, July 31). Guidelines for the practice of telepsychology. Washington, D.C.; APA. Recuperado de http://www.apa.org/practice/guidelines/telepsychology.

Gagliesi, P. (2020). Recomendaciones para terapeutas en los tratamientos online. DBT Latinoamérica. Recuperado de https:// www.facebook.com/pablo.gagliesi/posts/10160086344668312.

Macías M., J.J. y Valero A., L. (2018). La psicoterapia on-line ante los retos y peligros de la intervención psicológica a distancia. Apuntes de Psicología, 36(1-2), 107-113.

Organización Panamericana de la Salud (2020, 12 de marzo). La OMS caracteriza a COVID-19 como una pandemia. Recuperado de https://www.paho.org/hq/index.php?option=com_content\&view=article\&id=15756:who-characterizes-covid-19-as-apandemic\&Itemid=1926\&lang=es.

Reyes, M. (2020). Guías para la práctica de la telepsicología: entrenamiento, estándares éticos y requerimientos legales. [Actualización de estado]. Facebook. Recuperado de https:/www.facebook.com/MichelAReyesO/posts/147903416731375? tn_ $=\mathrm{K}-\mathrm{R}$. 